\title{
AN EFFECTIVE PROTECTION OF DATA FOR ORGAN DONATION USING BLOCKCHAIN TECHNOLOGY
}

\author{
Beena G Pillai \\ Assistant Professor, Department of Computer Science and Engineering, \\ GITAM - School of Technology, Bengaluru, India \\ Madhurya J A \\ Assistant Professor, Department of Computer Science and Engineering, \\ GITAM - School of Technology, Bengaluru, India

\section{Dr. Dayananda lal N} \\ Assistant Professor, Department of Computer Science and Engineering, \\ GITAM - School of Technology, Bengaluru, India

\section{Dr. Jeena Jecob} \\ Associate Professor, Department of Computer Science and Engineering, \\ GITAM - School of Technology, Bengaluru, India
}

\begin{abstract}
The health maintenance business is constantly viewing symbols of alteration in addition to behind innovative development as well as improvement. Nowadays crime is accomplished into the name of organ transplantation. High priority list for some specified blood group such as AB group, Bombay blood which was matching with some registered donors group to come up to the directed attention of criminals and murder them. The crime accomplished through the hospital intended for organ transplantation so that the criminals are supposed to not keep in the name of deficient in supported data. Several sets of laws for organ transplantation are pointing out which determine to prevent every species of fake organ transplantation. The killing of prospective donors on behalf of the sake of organ transplantation sting, on the background of sinking quantity of organ transplantation in different states in India all through current existence mostly suitable to severe commandments. One of the biggest supplies in these days' health care method is to defend the donor's medical report aligned with latent intruders. Therefore, it is essential to include protected data that can just support public preserve to get the donor's medical report. As a result, we have projected Blockchain technology as a distributed move toward precautions in right to use the remedial statement of a donor. It's cool, calm and collected of three stages 1 . Key Generation, 2. DES Encryption and 3. Information recovery via BlockChain
\end{abstract}


technology. This planned structure possibly will guarantee the security of the donors and additionally maintain the protection and responsibility of the healthcare system.

Key words: Blockchain, DES, Decryption, Encryption, Organ transplantation, Security.

Cite this Article: Beena G Pillai, Madhurya J A, Dr. Dayananda lal $\mathrm{N}$ and Dr. Jeena Jecob, An Effective Protection of Data for Organ Donation using BlockChain Technology. International Journal of Electrical Engineering and Technology, 11(5), 2020, pp. 73-82.

http://iaeme.com/Home/issue/IJEET?Volume=11\&Issue $=5$

\section{INTRODUCTION}

Donations to organs are allowed under Indian law. The Indian Government supported the 1994 Human Organ Transplantation Act that permits organ donation, and legally acknowledged the concept of "brain death." Brain death is the irrevocable and final completion of the brain mission. A person cannot prolong his or her own life in the circumstances of brain death, but critical body functions can be sustained for a short period in an intensive care unit. These persons are held back on artificial hold to retain organ oxygenation so that the organs remain in a stable condition before they are removed. Such patient's organs can be transplanted into terminally ill patients. Existing legislation decides that the family is responsible for the decision of organ donation after death. The reason for death and the ABO blood type was only recognized in the reports of the efficient donors, which made it impractical to correlate these variables with successful contribution along with suitable donors. Also, restrictions of the study consist of the deficiency of explicit objects in some of the BD rules not accomplished, the uncompleted contributions and are deficient in recognition of the causes for family rejection.

Generally, blockchain technology acts as the most important part of the health and healthcare organization. As a consequence of the decentralized and circulated technology, Blockchain affords protection into healthcare. Blockchain improvement agrees through the individual enhanced supervision in the direction of protected data distribution between different associates, information interoperability, flexible and quick charging. Nowadays, the tool has a quick development within its coming prospects among an extensive digital revolution via creating a better substitution day by day. Internet of things, identifying progression, and $5 \mathrm{G}$ is the rapidly increasing development providing markable obligations to individual refrain managements. The federal plan in present health care services is not so safe with the different health checkups, which presents an interruption in accessing the information with it, and has a foremost hazard in the outflow of data. In such a case, the health statements are recorded lacking the awareness of the donor. Accessing the information in a protective method contained by the system is the main problem in the existing health concern sustaining method. For accessing the statistics, Blockchain is the proficient method in addition to a guaranteed technology.

Nowadays organ donation systems are responsible for gathering information and organ transplantations have a lack of transparency. And it is usually slow as well as it is an existence aggressive substance. These methods are among the least amount of protection moreover through enhancement in current computer dispensation authority and algorithms. It is most excellent to get a careful come up to circumvent upcoming barriers. In recent times nearby an enhancement in safety violation, negotiating client confidentiality in addition to the reliability of the method. Contemporary arrangements hold moreover supervise records employing established databases; though the majority of hospitals, health ministries along with further 
health amenities accomplish not include consistent coordination for conversing statistics. Permanent medicinal services situations have to integrate the improving assignments commencing the victims as nicely as the experts in the radiance of the reality that the professional is similarly necessary to improve the beneficial documents to explore the precedent uplifting proceedings furthermore together with uplifting medicine or several exceptional consistent statements. Accordingly, our planned structure is through plus huge alert on such communal assurance circumstances wherever together with and improving responsibilities preserved be real concluded via the two patients presently as consultants.

\section{RELATED WORK}

Zibin Zheng et.al [1] Present an exhaustive description of blockchain. This is an analysis of blockchain technology regarding blockchain databases [7], as well as main blockchain functionality [14], [15]. The common consensus architectures used during blockchain are discussed and evaluated in distinct ways.

Mr Qiuyun Lyu et.al [2] has proposed a safe blockchain-based authentication methodology, known as SBAC, to ensure that rights holders can share review and revoke their information in such a secure manner. Ultimately, to accomplish decentralized security, [3], [10]-[13] and develop a corresponding framework and introduce a blockchain-based availability credential system to avoid system failure and preserve security with the review. The cuckoo filter is implemented for effective confirmation of control tokens demand. Also, our SBAC retains the attribute of omnipresent ICN optimization.

Mary Subaja Christo et.al [3] introduced Blockchain Technology as a deposited solution to providing protection when obtaining a people's clinical record. This proposed framework may also ensure patient protection and, besides, maintain the health care system's security and trustworthiness. The key core is to validate and acquire successful knowledge resources for today's social security systems using creativity in the squares network.

Diogo F. Pacheco et.al [4] It appears that organ-related social media interactions often appear to be correlated with elements of organ donation including such organ transplants similarities. [20]-[22] We have found differences in the different organs that are debated prominently for every geographic area and that these differences tend to be correlated with facets of organ donation in that area. And these differences tend to be linked to facets of organ donation within this province, for example, the particular quantity of kidney interactions in Kansas. The unusual amount of kidney interactions in Kansas, the results indicate that the suggested method is capable of characterizing organ donation understanding in real-time.

Electronic medical preparation moves towards the physical and distant right to use of health statements along with the confidentiality of the documentation are the majority of necessary pastures of the function wherever Blockchain technology preserves assessment. The health documentation in which a decentralized technique for exploited blockchain automatic ability is established to the agreement through the EHR/EMR and additionally provide a prospective appropriate investigation of blockchain procedure in public assurance, which provides a model to EHR/EMR. Furthermore, medical share provides the hopeless technique for distributing the experimental statements with a variety of professional associations exploiting blockchain. Consequently, the assessment system distinguishes the outstanding organizations for accessing the proceedings securely operating blockchain improvement. 


\subsection{Objectives}

- To provide blockchain Security for the organ donation healthcare system.

- Protection characteristics by maintain privacy, reliability and verification of donor information and to recognize the protection consequence of blockchain

\section{METHODOLOGY}

\subsection{Basic Approach}

Nowadays fault is accomplished in the name of organ transplantation. High priority list for some specified blood groups such as $\mathrm{AB}$ group, Bombay blood which was matching with some registered donors assembly to approach below the focal point of the criminals and killing them. The crime accomplished through the hospital for organ transplantation consequently that the criminals are hypothetical to not be accumulated in the name of is deficient in of substantiation. Several regulations for organ transplantation are bringing up which will discontinue the entire varieties of fake organ transplantation. The assassination of possible donors intended for the sake of organ transplantation sting, on the environment of dropping statistics of organ transplantation in special states in India all through latest existence essentially suitable to exacting laws. A most important concern in organ transplantation is the description of death and predominantly brain death. An additional most important significant feature is the conjugal susceptibility of an explicit evolution to contribute to organs. From the transfusion position of analysis, uncommon blood is the one which is deficient in soaring reliability antigens as well as the one who lacks several regular antigens and such blood donations assist in transfusion to those beneficiaries having all antibodies to equivalent antigens. In India, we enclose regarding four such varieties of phenotypes possible adequate to pretence troubles in giving that blood to the beneficiaries having these phenotypes. Next to, nearby additional four kinds of uncommon drops of blood that pretend infrequently predicaments in the blood contribute, although several of these may perhaps reason predicaments in recognizing consequences on transporting suitable blood groups for a person.

\subsection{Overview of Working Flow}

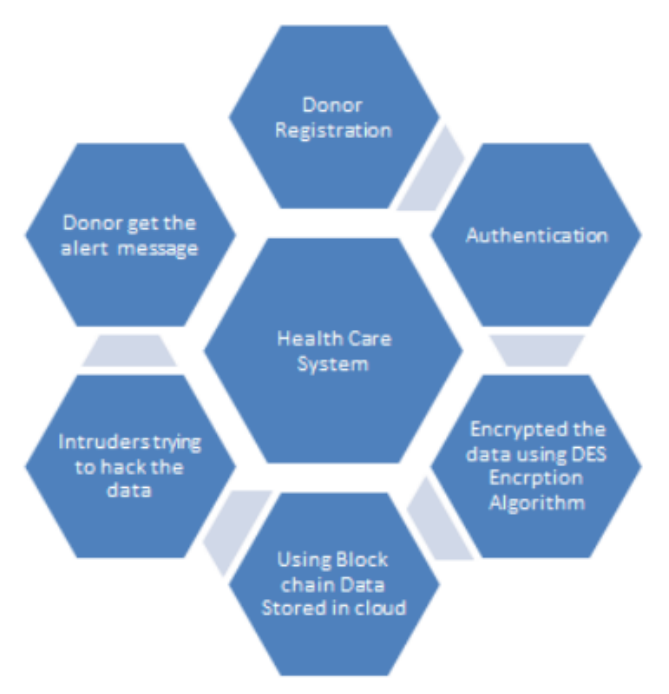

Figure 1(a) Work flow of Organ Donation healthcare system - stage1 
The donor is a new user so every personal detail will be registered in a separate account. The reorganized health statement is encrypted using the Data Encryption Standard (DES) algorithm and the encrypted information is accumulated in the confidential cloud, wherever we can recognize the site effortlessly. Concentrate on the encrypted information in the confidential cloud is hoard in the blockchain.

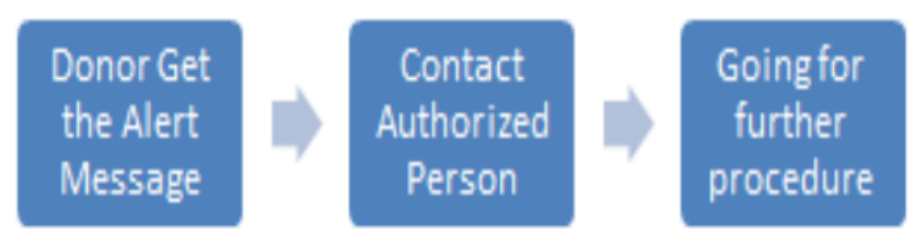

Figure 1 (b) Work flow of Organ Donation healthcare system - stage2

The administration department keeps the record of all donors who are registered. But for them also the donor details are not able to be accessed. If somebody is trying to access the information the corresponding message is getting into the donors mobile as well as email. If it is unethical, then the donor can make a complaint against this department. Using blockchain technology, the confirmation is carried out to check the legal person who requests to monitor the donor's medical report. The authoritative person preserves to append or recover the health statement through the donor's consent. However, he/she cannot transform the donor's health record.

\section{SUGGESTED SYSTEM}

Blockchain technology as a distributed move toward more protection when hacking a donor's medicinal information. It is a collection of three 1. Key Generation 2. DES Encryption and 3. DES Decryption using BlockChain technology. A 64bit key is used to input the algorithm for key generation and is transformed into the 56-bit key. A different 48bit subkey is generated from the 56bit key and is called key transformation, for DES Encryption a block is encrypted into an initial permutation IP, then into a composite key-dependent computation, and in conclusion into a permutation reversed from the initial permutation IP, then to a complex keydependent computation and finally to permutation which is inverse of the initial permutation IP.

\subsection{Registration of a New Donor}

Generate new Id for the donor.

The Kerberos software is an implementation of client-server enabling secure transfers through connections. The company provides good security for customers, as well as honesty and privacy. Authentication preserves real identities of most of the transmitter and the recipient of a system transaction. 


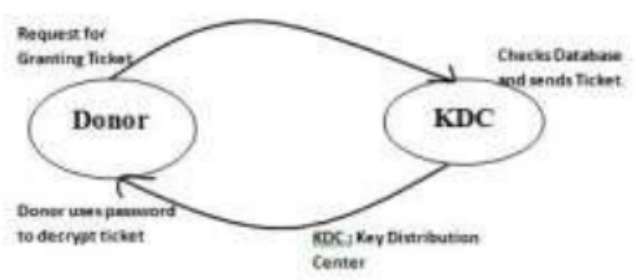

Figure 2 Registration of New user

\subsection{DES Encryption}

It's proceeding via three stages,

1. Initial permutation: 64 bits of block to be encrypted and subjected to permutation.

2. Key-based Computation: Let 64 bits of the input block in an expansion consisting of a $32-$ bit block L preceded by 32-bit block R. Let K become the 48-bit block selected from the 64bit key.

$$
\begin{aligned}
& L^{\prime}=R \\
& R^{\prime}=L(+) f(R, K) \\
& \text { When ' } n \text { ' is in the } \\
& \text { range, } \\
& \text { Then } L_{n}=R_{n-1} \\
& R_{n}=L_{n-1}(+) f\left(R_{n-1}, K_{n}\right) T
\end{aligned}
$$

3. Key schedule: Let KS be a process that describes an integer $\mathrm{n}$ in the ranging from 1 to 16 and a 64-bit block key as input and produces a 48-bit block kn as output that is permitted to pick bits through the key.

$$
\mathrm{K}_{\mathrm{n}}=\mathrm{KS}(\mathrm{n}, \mathrm{KEY})
$$

Now, information recovery preserve is executed only by the certified person. After verification, the person can accept the hash price of the encrypted information. And the IP1 permutation implemented to the pre-output block for DES decryption is the contrary of the original IP permutation added to the input. To decipher it is only appropriate to put on this very same algorithm to an enciphered block of messages, taking note that the same block of the key bits $\mathrm{K}$ is used in decipherment at each iteration of the procedure.

\subsection{Data Recovery}

$\mathrm{R}_{10} \mathrm{~L}_{10}$ as the permuted input, $\mathrm{K}_{10}$ is used in the first iteration, $\mathrm{K}_{10}$ is the second iteration and so on, with $\mathrm{K}$ is used in the $16^{\text {th }}$ iteration. The information is decrypted via the DES decryption. Hence, the health statement of the donor is protected by blockchain technology. 


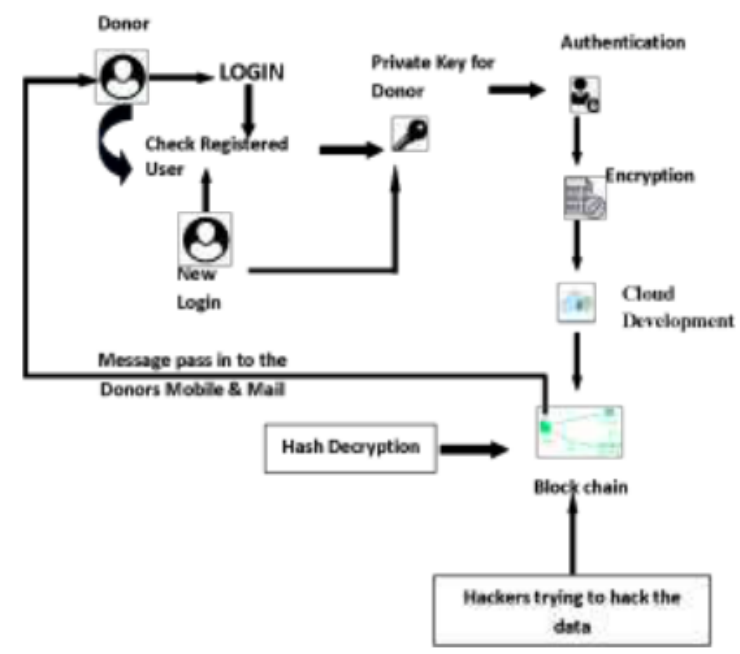

Figure 3 Information stored in cloud and data recovery

\section{RESULT \& DISCUSSION}

The donors who are interested to donate their organs after death register into the health organization. The details of the donors are hidden from others. All the details are filled in a particular format and saved in the cloud. Anybody trying to access the data at the time itself a message sent to the donor's registered mobile number and registered mail. After the death of the donor health department access the data, a private key generated and sent to the donor mobile number. Then using this private key department can access the data then they know about the donors' details regarding blood group as well as whichever the organs are registered for donation. Electronic Medical Record is the present online healthcare examinations which take part in a significant function in observance and accumulating the information, which has the main concern in the outflow of donor's information.

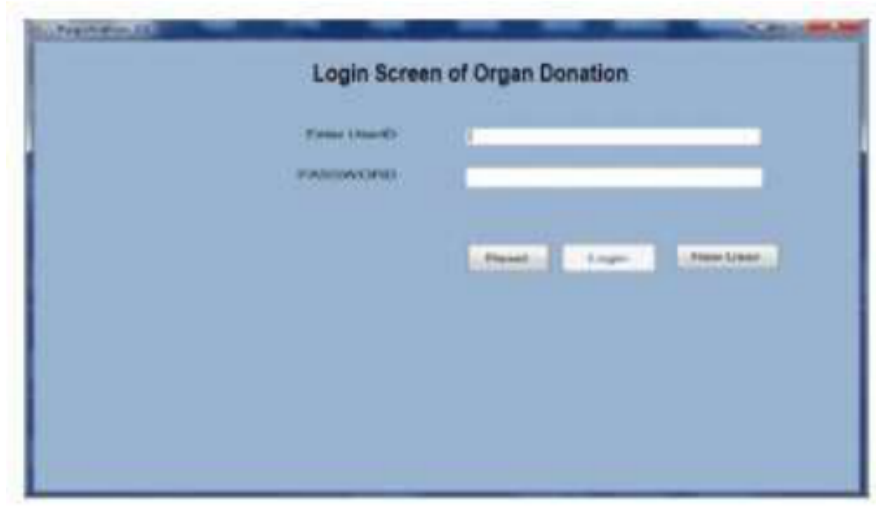

Figure 4 Current User Registration

In blockchain expertise, the information is hoarded as ledger attributes which preserve and observe the intruders in capturing the health documentations. These become the most important motive in support of the growth of Blockchain technology. In Blockchain technology, not only afford protection and convenience, however to provide additional manufacturing fundamentals in the supervision and follows confidentiality, morality, and authentication. Thus the foremost intent of this investigation is to afford protected administration in admitting the health verifications via blockchain technology through exclusive recognition of the information safety. 


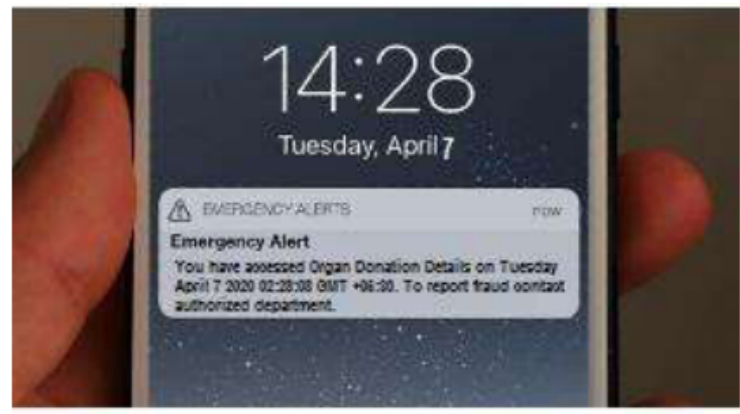

Figure 5 Security Alert Message

Security: The donor information is more secure and its hide from the intruders.

Scalability: Given that it is a confidential open area sequence, the time taken for the entire process is less significant than the major coordination. It is predictable through the response point of each replacement. The outcome pointed to as the usual occasion for the age of one more squarely is approximately 13 secs.

Reliability: Morality presumes an essential work in receiving the beneficial description of a donor. Because the donor is efficiently selected by the professional, none of the other people can modify/adjust the information. In our circumstances, the stranger can't preserve the opportunity to adjust or adjust the restorative report of a donor.

Access control: Access control selections specified through a donor. Lying on the off possibility to facilitate the stranger person desires in the direction of the beneficial statement, next to spot it relies on the donor's approval usually precipitately split ends the outsiders ask.

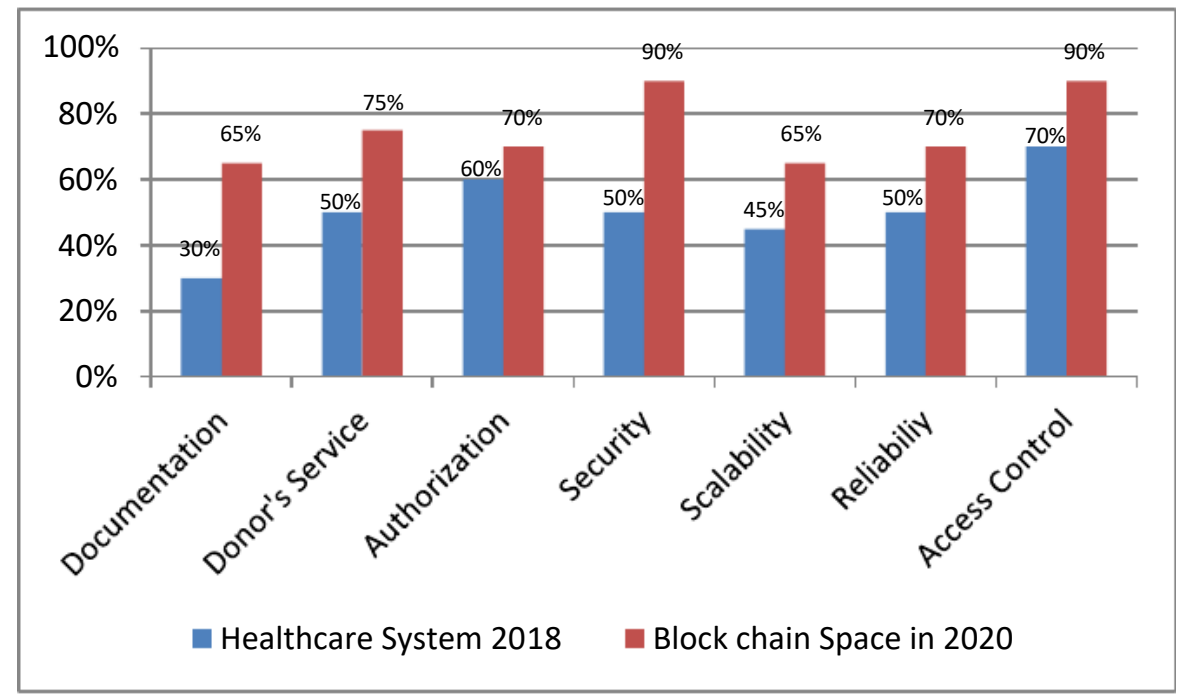

Figure 6 Secure Document Transfer: Electronic Hospital Records

Blockchain records all the transactions that take place on the network. System services like a web server. This will run the backend process. The proposed system authentication with public key cryptography to ensure people and devices are authenticated and identified securely. The records are protected through cryptography on a blockchain. It is considered reliable because full copies of the block- chain ledger are maintained by all active nodes.

\section{CONCLUSION}

Blockchain in remedial services constructions has massive unwrap accesses. It creates a prospective extent in the communal assurance dealing for a variety of followers giving secure 
and productive data. Organ donation system, authenticate and valuable data gets to implement designs for current public assurance frameworks that make use of blockchain improvement. Additionally, we considered that our projected preparation would be able to assure the basics of responsibility, confidentiality and confirmation in this organ transplantation examination.

\section{REFERENCES}

[1] Zibin Zheng, Shaoan Xie, Hongning Dai, Xiangping Chen, and Huaimin Wang (2017) “An Overview of Blockchain Technology: Architecture, Consensus, and Future Trends" IEEE 6th International Congress on Big Data

[2] Qiuyun Lyu, Yizhen Qi, Xiaochen Zhang, Huaping Liu, Qiuhua Wanga, Ning Zheng (2020) "SBAC: A secure blockchain-based access control framework for information-centric networking" Journal of Network and Computer Applications 149, 102444

[3] Mary Subaja Christo, Anigo Merjora A, Partha Sarathy G, Priyanka C and Raj Kumari M (2019)"An Efficient Data Security in Medical Report using BlockChain Technology" International Conference on Communication and Signal Processing, April 4-6, India

[4] Diogo F. Pacheco, Diego Pinheiro, Martin Cadeiras and Ronaldo Menezes (2017) "Characterizing Organ Donation Awareness from Social Media" IEEE 33rd International Conference on Data Engineering

[5] Christo MS, Meenakshi S. (2016) Enhancing security properties of Rumor Riding protocol under various attacks scenario in the P2P network. In2016 International Conference on Communication and Signal Processing (ICCSP) Apr 6 (pp. 1130-1135).

[6] Christo, M.S. and Meenakshi, S., (2018). Enhancing Rumor Riding protocol in P2P network with Cryptographic puzzle through challenge question method. Computers \& Electrical Engineering, 65, pp.122-138.

[7] F. Jabeen, Z. Hamid, A. Akhunzada, W. Abdul, and S. Ghouzali, (2018) "Trust C. Esposito, A. D. Santis, G. Tortora, H. Chang, and K. K. R. Choo, "Blockchain: A panacea for healthcare cloud-based data security and privacy?” IEEE Cloud Computing, vol. 5, no. 1, pp. 31-37, Jan

[8] S. P. Bingulac, (1994) "On the compatibility of adaptive controllers (Published Conference Proceedings style)," in Proc. 4th Annu. Allerton Conf. Circuits and Systems Theory, New York, pp. 8-16.

[9] P. Zhang, M. A. Walker, J. White, D. C. Schmidt, and G. Lenz, (2017) "Metrics for assessing blockchain-based healthcare decentralised apps," in 2017 IEEE 19th International Conference on e-health Networking, Applications and Services (Healthcom), Oct, pp. 1-4.

[10] M. Mettler, (2016) "Blockchain technology in healthcare: The revolution starts here," in 2016 IEEE 18th International Conference on e-Health Networking, Applications and Services (Healthcom), Sept, pp. 1-3.G

[11] Anthem, doc.ai launch healthcare AI data trial on the blockchain. In: HITInfrastructure [Internet]. 6 Aug 2018 [cited 6 Dec 2018]. Available: https:// hitinfrastructure.com/news/anthem-doc.ai-launch-healthcare-ai-datatrial- on-blockchain.

[12] Melendez S. (2018) How IBM and the CDC are testing blockchain to track health issues like the opioid crisis. In: Fast Company [Internet]. 4 Sep [cited 5 Dec 2018]. Available: https://www.fastcompany.com/ 90231255/how-ibm-and-the-cdc-are-testing-blockchain-totrack health- issues-like-the-opioid-crisis.

[13] Why blockchain could transform the very nature of EHRs. In: Healthcare IT News [Internet]. 18 Sep 2017 [cited 6 Dec 2018]. Available: https:// www.healthcareitnews.com/news/whyblockchain-could-transformvery- nature-ehrs. 
[14] The truth about blockchain. In: Harvard Business Review [Internet]. 1 Jan 2017 [cited 6 Dec 2018]. Available: https://hbr.org/2017/01/the-truth-aboutblockchain.

[15] World Health Organization, Patient Safety: Making Health Care Safer, (2017) Available at: http://www.who.int/patientsafety/publications/patient-safetymaking- health-care-safer/en/. Accessed January/20, 2018.

[16] H. Campbell, R. Hotchkiss, N. Bradshaw, M. Porteous, (1998) Integrated care pathways, BMJ 10 (January (316)) 133-137 7125.

[17] I. Scott, (2009) What are the most effective strategies for improving quality and safety of health care? Intern. Med. J. 39 (June (6)) 389-400.

[18] R.J. Coffey, J.S. Richards, C.S. Remmert, S.S. LeRoy, R.R. Schoville, P.J. Baldwin, (1992) An introduction to critical paths, Qual. Manag. Health Care 1 (1) 45-54 Fall.

[19] M.F. Aarnoutse, S. Brinkkemper, M. de Mul, M. Askari, (2018) Pros and cons of clinical pathway software management: a qualitative study, Stud. Health Technol. Inform. 247, 526530.

[20] L. Kinsman, T. Rotter, E. James, P. Snow, J. Willis, (2010) What is a clinical pathway? Development of a definition to inform the debate, BMC Med. 27 (8) (May) 31- 7015-8-31. 